\title{
Surgical Candidacy in Skull Base Paragangliomas: An Institutional Experience
}

\author{
Govind Bhuskute ${ }^{1, \odot}$ Ravi Sankar Manogaran ${ }^{1, \odot} \quad$ Amit Keshri $^{1} \quad$ Anant Mehrotra $^{2} \quad$ Neha Singh $^{1}$ \\ Arulalan Mathialagan ${ }^{1}$ \\ ${ }^{1}$ Neuro-otology Unit, Department of Neurosurgery, Sanjay Gandhi \\ Post Graduate Institute of Medical Sciences, Lucknow, \\ Uttar Pradesh, India \\ 2Department of Neurosurgery, Sanjay Gandhi Post Graduate \\ Institute of Medical Sciences, Lucknow, Uttar Pradesh, India

\begin{abstract}
Address for correspondence Ravi Sankar Manogaran, MS (ENT), DNB, Neuro-otology Unit, Department of Neurosurgery, Sanjay Gandhi Post Graduate Institute of Medical Sciences, Lucknow 226014, Uttar Pradesh, India (e-mail: drravisankarpgi@gmail.com).
\end{abstract}

J Neurosci Rural Pract:2021;12:116-121

\begin{abstract}
Keywords

- carotid body tumor

- vanillylmandelic acid

- angiography

- bilateral skull base

- vagal paraganglioma

Objective The aim of the study is to determine the surgical candidacy and nuances of skull base paraganglioma surgery in the era of radiotherapy.

Materials and Methods This was a retrospective observational study conducted in patients who presented with skull base paragangliomas between January 2017 and December 2019. Primary data, including indication for surgery, the approach used, the extent of resection, complications, and postoperative lower cranial nerve status were studied.

Results A total of 21 cases of skull base paragangliomas were analyzed, including seven cases of tympanic paraganglioma, 10 cases of jugular foramen paraganglioma, three cases of multiple paragangliomas, and one case of vagal paraganglioma. Indications for surgery were young age, bleeding from ear, neck mass with upper aerodigestive pressure symptoms, lower cranial nerve paralysis, and patients with intracranial pressure symptoms. Total excision was done in 11 patients, near-total excision in five patients, subtotal in three patients, and surgery was not done in two patients. Facial nerve paralysis was the most common complication observed, followed by bleeding and flap necrosis. Radiotherapy was considered as adjuvant treatment wherever indicated.

Conclusion A thorough knowledge and understanding of the pathophysiology of the skull base paragangliomas and its management strategies can help to achieve excellent results in terms of tumor clearance and reduction in complications. A multidisciplinary team approach and meticulous skull base surgical techniques have a significant role to play in the management of paragangliomas, especially in developing countries where availability of radiosurgery is still a challenge.
\end{abstract}

\section{Introduction}

Skull base paragangliomas (PGs) are neural crest tumors, which originate in the parasympathetic system in relation to the neurovascular bundle. They are a group of rare neoplasms which account for $0.6 \%$ of all head and neck neoplasms. ${ }^{1}$ PG, although slow-growing and benign, tend to local destruction and intracranial spread which may affect the vital neurovascular structures of the skull base. Multicentric PGs have an

DOI https://doi.org/

$10.1055 / \mathrm{s}-0040-1721201$

ISSN 0976-3147. incidence of 10 to $20 \%{ }^{2}$ Succinyl dehydrogenase mutation is associated with multicentricity, be it familial or sporadic ${ }^{3}$ or malignant variant of PG.

There are multiple staging systems to classify the spread of the disease of which Glasscock Jackson ${ }^{4}$ and Modified Fisch $^{5}$ classification are used for tympanojugular PG, Shamblin's staging $^{6}$ for carotid body PG, and Netterville staging system ${ }^{7}$ for vagal PG.

(c) 2021. Association for Helping Neurosurgical Sick People.

This is an open access article published by Thieme under the terms of the Creative Commons Attribution-NonDerivative-NonCommercial-License, permitting copying and reproduction so long as the original work is given appropriate credit. Contents may not be used for commercial purposes, or adapted, remixed, transformed or built upon. (https://creativecommons.org/licenses/by-nc-nd/4.0/) Thieme Medical and Scientific Publishers Pvt. Ltd., A-12, 2nd Floor, Sector 2, Noida-201301 UP, India 
Thorough preoperative work-up is essential in deciding the treatment modalities. Importance of history taking and clinical-neurological examination cannot be subdued. Imaging modalities like computed tomography (CT) and magnetic resonance imaging (MRI) are mandatory to diagnose the disease and define its anatomical extension and relation to vital neurovascular structures. Preoperative digital subtraction angiography (DSA) with embolization of feeding vessels can be done in selective patients considered for surgery. Twenty-four-hour urinary vanillylmandelic acid (VMA) and ultrasonography of abdomen should be done to rule out multicentricity and functional tumors.

The current modalities of managing skull base PGs are surgery, radiotherapy, embolization, and active observation. Of these, embolization does not have a significant role in disease control and is mostly employed as an adjuvant to surgery. Because of slow-growing nature, an observation by periodic surveillance can be considered as a treatment modality after discussing it with the patient ${ }^{8}$

Age, comorbidities, tumor type and stage, multicentricity, the functionality of cranial nerves, hearing status, venous drainage of the brain, and the degree of carotid involvement are critical factors that affect decision making. ${ }^{9}$ In young patients, the emphasis is to cure the disease rather than controlling it. The objective is to avoid long-term complications of the disease as well as the treatment modality.

Radiotherapy mainly aims for disease control rather than cure, and it has its rightful advantage of little to no immediate neurovascular complications. However, its practical application in developing countries is a challenge where its availability, financial burden in low socioeconomic group, and patient follow-up pose difficulty. In contrast, with meticulous patient selection and a well-trained surgical team, skull base surgery in selected cases has more benefits in terms of having tumor-free survival.

In this paper, we study the candidacy for surgery and its outcome managed in a tertiary care institute situated in India.

\section{Materials and Methods}

This was a retrospective observational study conducted in the neurosurgery department of a tertiary referral center. Approval by Institute of Ethical Committee was obtained.

Medical records of patients with a definitive diagnosis of skull base PGs (tympanojugular PG, vagal PG, and carotid body PG) from the year 2017 to 2019 were obtained. These patients had undergone a thorough clinical examination to look for local and regional spread of the disease. All patients underwent preoperative CT and MRI and 24-hour VMA testing. Those with advanced stages were subjected to DSA and preoperative embolization.

The patients were explained about all the available treatment modalities and their advantages and potential intra and post-treatment complications. These patients were managed by either surgery, radiotherapy, combined modalities, or wait and scan approach. Patients with subtotal excision were further considered for radiotherapy.
Criteria used for surgery as the primary treatment modality for:

1. Tympanic PG

2. Jugular foramen PG in

i. Young patients with or without lower cranial nerve palsy.

ii. Patients with bleeding aural mass.

iii. Patients with lower cranial nerve palsy.

iv. Patients with intracranial pressure symptoms.

3. Vagal PG in

i. Young patients.

ii. Patients with upper aerodigestive pressure symptoms.

4. Carotid body PG-Shamblin's stage 1 and 2.

5. Multicentric tumors with functional lower cranial nerves on at least one side.

6. Malignant PG.

\section{Results}

Twenty-one patients of skull base PGs were studied. Of these, nine $(42.8 \%)$ were males, and $12(57.1 \%)$ were females. The average age at presentation was 41.6 years, the youngest patient was of 19 years, whereas the oldest was 62 years old. Clinical features at presentation were hearing loss (81\%) followed by tinnitus (62\%), bleeding from the ear (43\%), facial nerve palsy (29\%), and lower cranial nerve palsy (38\%) (-Table 1 ).

Table1 Symptomatology

\begin{tabular}{|l|l|}
\hline Clinical features & Percentage of patients \\
\hline Hearing loss & $81 \%$ \\
\hline Tinnitus & $62 \%$ \\
\hline Bleeding from ear & $43 \%$ \\
\hline Lower cranial nerve palsy & $43 \%$ \\
\hline Facial nerve palsy & $29 \%$ \\
\hline Intracranial pressure symptoms & $9 \%$ \\
\hline
\end{tabular}

Total excision of the tumor was considered when gross total excision of the tumor was done using an operating microscope, and no residual tumor on follow-up imaging. Near-total excision was when follow-up imaging had a minimal residual tumor. Subtotal excision of the tumor was considered when less than $50 \%$ of the tumor was excised.

\section{Tympanic Paraganglioma}

There were seven patients of class B tympanic PG. The most frequent complaints were hearing loss (100\%), tinnitus (71\%), and aural bleeding mass (57\%). One patient had preoperative grade IV (House-Brackmann [HB] classification ${ }^{10}$ ) facial nerve palsy. Six of these patients underwent surgery. One patient refused surgery and was kept under regular follow-up. Using the transmastoid approach, five patients underwent total excision of the tumor, and one underwent near-total excision. Postoperatively one patient had grade IV (HB) facial nerve palsy, which recovered within 3 months of surgery. 
One patient had suture site abscess, which was managed with oral antibiotics while another patient had dehiscence of cul-de-sac, 1 year after surgery (-Fig. 1).

\section{Jugular Foramen Paraganglioma}

There were 10 patients of jugular foramen PGs. All patients had advanced disease at presentation with seven having lower cranial nerve palsies, five with bleeding aural mass, and one patient with raised intracranial pressure symptoms at presentation (-Table 1). All patients were managed with surgery, where total excision of the tumor in three patients, near-total excision in four patients, and subtotal excision in three patients were done. Among the patients who underwent near-total and subtotal excision, four of them were subjected to postoperative radiotherapy while the rest three patients were managed with
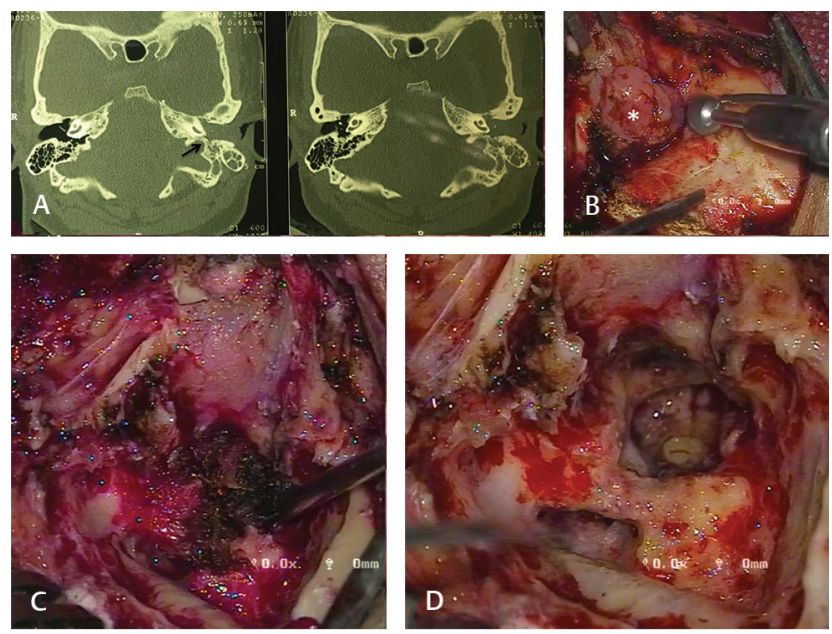

Fig. 1 Tympanic paraganglioma. (A) High resolution, noncontrast computed tomography of temporal bone in axial plane showing mass filling the external auditory canal. It can also be noted that the jugular bulb appears intact (arrow). (B) Pinkish, lobulated, vascular mass filling the external auditory canal (asterisk). (C) Gradual debulking of the tumor being done. (D) View of site after completion excision of tumor. regular follow-up imaging. No patient developed new-onset lower cranial nerve palsy. One patient with intracranial pressure symptoms had undergone decompression via retro sigmoid approach and subsequently developed meningitis on the third postoperative day and succumbed to it by day 10 . Three patients developed flap necrosis which improved subsequently without any active intervention (-Fig. 2).

\section{Vagal Paraganglioma}

There was one patient with vagal PG who had preoperative ipsilateral vagal palsy with neck swelling and upper aerodigestive pressure symptoms due to oropharyngeal bulge. Total excision of the lesion was done. The tumor extended up to the inferior ganglion of the vagus (Nettervile stage two). All other lower cranial nerves were anatomically preserved. Postoperatively along with the vagal palsy, patient developed IX, XI, and XII cranial nerve palsy (-Fig. 3).

\section{Multiple Paraganglioma}

There were three patients with PG at multiple sites. One patient had bilateral carotid body tumors with bilateral pheochromocytoma. The endocrine surgeons surgically managed pheochromocytoma before excising the carotid body tumors. The patient developed recurrence of the right carotid body tumor and is currently being actively observed with yearly follow-up. Another patient had ipsilateral carotid body tumor (Shamblin 1) with jugular PG (Di2) who presented with hearing loss and lower cranial nerve palsies and intracranial pressure symptoms. Both the tumors were managed simultaneously. Carotid body tumor was excised in toto whereas subtotal excision was done for jugular PG as there was excessive blood loss. One patient had bilateral jugular PGs with bilateral pheochromocytomas. The patient had undergone surgery for pheochromocytoma while surgery is planned for jugular foramen $\mathrm{PG}$.
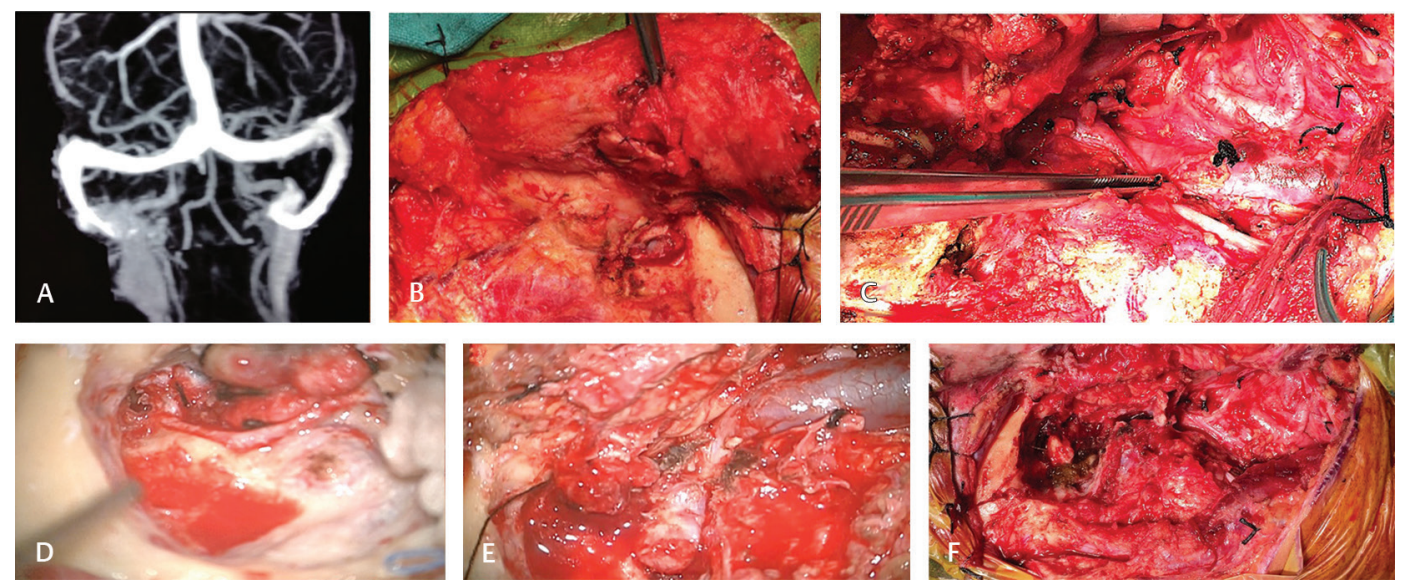

Fig. 2 Jugular foramen paraganglioma managed by Fisch-Type A postauricular transtemporal approach. (A) Magnetic resonance venogram image showing filling defect in right jugular foramen extending into the internal jugular vein (IJV). (B) Anteriorly based skin flap elevated and cul-de-sac closure was done. (C) Neck vessels and lower cranial nerve courses were clearly identified. (D) Tympanic and mastoid segment of facial nerve was decompressed after canal down mastoidectomy followed by anterior transposition. (E) Sigmoid sinus, jugular bulb, IJV course clearly delineated. (F) Cavity after complete tumor removal with preservation of medial wall of jugular bulb. 

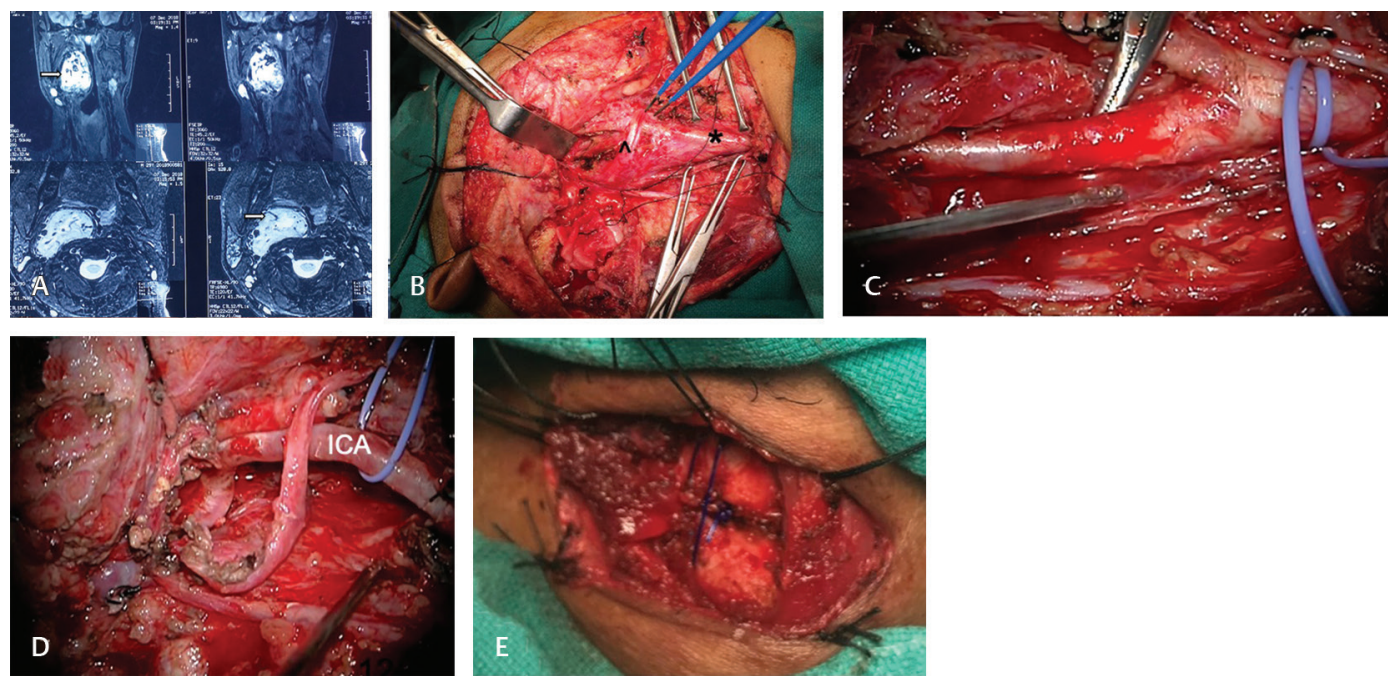

Fig. 3 Vagal paraganglioma. (A) Magnetic resonance imaging in coronal and sagittal planes showing hyperintense lesion filling the right parapharyngeal space extending into the neck (arrow). (B) Exposure of the tumor via transcervical approach. Asterisk $\left({ }^{*}\right)$ : common carotid artery, top arrow $\left({ }^{\wedge}\right)$ : hypoglossal nerve. (C) Internal carotid artery dissection. (D) Cavity after tumor excision with anatomically preserved 11,12 nerves. (E) Right side cricothyroid approximation.

\section{Discussion}

Skull base PGs are rare forms of head and neck neoplasms. It is commonly encountered in fourth to fifth decade of life. In our series, the mean age at presentation was 41.6 years, and most of the patients were in an advanced stage of the disease. The youngest patient was 19 years old and presented with a jugular foramen PG. Rarely, PG has been diagnosed even in the pediatric age group. ${ }^{11}$

Carotid body tumors are most prevalent (60\%) amongst all the skull base PGs, followed by jugulotympanic PGs (35\%) with vagal PGs being the least common $(<5 \%) .{ }^{8}$ The incidence of multicentricity is 10 to $20 \%$ and is often associated with the familial predisposition. These tumors tend to be more aggressive and show high rates of recurrence. ${ }^{2}$ In our series, jugular foramen PG was $47 \%$ of all cases, followed by tympanic PG (33\%), multicentric PG (14\%), and a single case of vagal PG.

Tympanic PG arises from the tympanic branch of glossopharyngeal nerve situated on the promontory of the middle ear and has limited middle ear cleft involvement, however, can rarely extend intracranially via the carotid canal. Surgery is the treatment of choice in these tumors because of their surgical accessibility as they are usually away from the critical structures making complete excision possible without any added postoperative morbidity in most of the cases. In a study by Forest et al, 80 cases of tympanic PG with total excision done in 75 cases, recurrence was seen in only one case after 14 years of surgery. ${ }^{12}$ Spector et al in a review of 46 cases concluded that surgery is the best treatment of choice in these tumors. ${ }^{13}$ In our series complete excision was achieved in five of six patients. Tympanic PG with facial nerve palsy is rare. We had one patient who had grade IV HB facial nerve palsy at presentation. In this situation, it is necessary to emphasize the role of surgery in PGs. Unless there is gross involvement of facial nerve, a routine facial nerve decompression often helps to regain its function, and if the nerve sheath is involved, resection of the segment with nerve grafting can be done in the same sitting. In our patient, post total tumor excision, a complete facial nerve decompression was done following which the nerve function improved. In one patient with B3-staged lesion, surgery had to be abandoned after excision of almost $90 \%$ of the tumor due to excessive blood loss of the tumor. Radiotherapy can be reserved for patients who are elderly, unfit for surgery with extensive disease. ${ }^{14}$

Surgical resection of vagal PG causes vagal nerve palsy. In a systematic review of vagal PG by Suárez et al, vagal palsy was seen in $95.7 \%$ of the cases. ${ }^{15}$ When the vagal nerve palsy develops secondary to the disease, vocal cord compensation is adequate to prevent aspiration. Thus, it is ideal to wait and let the vagal palsy set in, following which the patient can be taken up for definitive treatment. Vagal PG can be accessed via various routes-transcervical, trans-submandibular, transcervical with mandibulotomy. The decision of which route to use depends on the size of the lesion and its relationship with the internal and external carotid artery. In our case, the indication for surgery was a young age, presence of vagal palsy, difficulty in swallowing, and breathing because of the oropharyngeal bulge. This patient underwent total excision of the tumor via transcervical route, which was extending up to the jugular foramen. All cranial nerves were anatomically preserved, but he had postoperative lower cranial nerve palsies (CN IX, X, XI, XII). He was managed with a temporary tracheostomy for aspiration and permanent cricothyroid approximation for improved speech and swallowing function.

Multiple PGs should be suspected in young patients with a positive family history. Selection of treatment modality should be individualized to each patient depending on the site and extent of the disease. In multiple ipsilateral PGs (e.g., jugular foramen and carotid body or vagal PG) attempt can be made to address both the lesions in the same sitting. Conservative approach should be considered in bilateral skull base disease. The decision of which side to operate first 
should be considered after assessing the intracranial vascular supply and lower cranial nerve status. Al-Mefty $\mathrm{O}$ et al recommended that in bilateral skull base lesions, lesions of smaller size and less probability of causing lower cranial nerve palsy should be operated first and only if the first surgery does not cause lower cranial nerve palsy then contralateral side should be operated. ${ }^{16}$ If there is a possibility of injury to lower cranial nerves, a cuff of tissue should be preserved along the nerves. These patients can then be subjected to adjuvant radiosurgery. We had three cases of multiple PGs. Those with ipsilateral lesions were managed in the same surgical setting. In bilateral carotid body lesions, we preferred surgery on the side with smaller sized lesion first.

Management of jugular foramen PG has remained a source of debate. In a series of 75 cases by Makiese et al, 59 cases underwent total excision of the tumor, 10 underwent near-total excision while six underwent subtotal excision. ${ }^{17}$ The most common complication encountered was iatrogenic lower cranial nerve palsies (18.7\%), facial nerve palsy (6.6\%), cerebrospinal fluid leak $(5.3 \%)$, and death (2.2\%). In another series of 53 cases of jugular foramen PGs by Sanna et al, where surgery was staged in far advanced stages of disease, ${ }^{18}$ total excision of the tumor was done in 49 patients and staged tumor removal was done in eight patients. Of these, $26.8 \%$ developed new-onset lower cranial nerve palsy; one patient had new-onset facial nerve palsy while two patients had cerebrospinal fluid (CSF) leak. Sanna et al and Makiese considered surgery as a primary modality of treatment in patients fit to undergo the procedure. In the series by Sanna et al, surgery was even considered in advanced disease (Di, V) in a staged manner. In a series by George Wanna on 12 patients with advanced disease (Glasscock Jackson class 3 and 4) with intact lower cranial nerve function, he suggested that leaving a cuff of tissue around the intact cranial nerves with postoperative radiotherapy prevents unnecessary neurological morbidity and also ensures tumor control. ${ }^{19}$

Both surgery and radiotherapy play a role in the management of Jugular foramen PG. ${ }^{20}$ However, there are specific indications where surgery is considered as the treatment of choice-young patients (irrespective of lower cranial nerve status), patients with bleeding aural mass, lower cranial nerve palsy at presentation, and in those with intracranial pressure symptoms. In our series of 10 patients, there were no new-onset lower cranial nerve palsies while two patients had new-onset facial palsy of which one recovered to grade III after 4 months of surgery. One patient who underwent decompression surgery for intracranial extension had CSF leak and developed meningitis on the third postoperative day following which he developed sepsis and died.

With the advent of radiosurgery, there has been significant debate on deciding treatment modality, especially in cases of jugular foramen PG. It is essential to achieve cure rather than tumor control in those who have more than 30 years of life expectancy. ${ }^{9}$ If radiosurgery is considered in young, its long-term effect is not known, and there may be a sudden increase in the size of tumor many years after radiosurgery. Hence, we prefer to advocate surgery in this group of patients. In patients with preoperative lower cranial nerve palsy, the neurological sequelae have already set in, and there is adequate compensation from contralateral vocal cord preventing aspiration and stridor. In such patients, radiosurgery has no role other than preventing a further increase in the size of the tumor. Also, in cases where there is a significant intracranial extension of disease, radiosurgery is not indicated as it can cause edema and it raised intracranial tension. In these cases, staged surgery often helps to achieve near-total to total excision of the tumor without added mortality or morbidity. The widespread availability of radiotherapy (especially radiosurgery) is still a challenge in developing nations. It requires periodic patient follow-up and imaging which adds a significant financial burden on patients.

The most grievous complications of surgery are meningitis and lower cranial nerve palsy. These have been extensively discussed in the literature. Apart from this one crucial complication that we encountered was postoperative flap necrosis. Four patients developed necrosis of skin flap postoperatively, despite anatomically preserving the arterial supply of the region. On further evaluation, we reviewed the DSA and embolization films which revealed the embolization of the postauricular artery, which is one of the major supplies of this flap.

Postoperative rehabilitation is necessary to improve the quality of life in these patients and should not be overlooked. Physiotherapy, speech, and swallowing therapy should be advocated in patients with lower cranial nerve palsy. In patients with uncompensated vagal palsy, we advocate doing cricothyroid approximation in the same sitting after tumor removal or during the follow-up. We had two such cases where cricothyroid approximation was done in the follow-up period. In literature, the cricothyroid approximation is shown to increase the tension on the paralyzed cord and partially restores horizontal vocal cord position, which increases the voice quality and swallowing outcomes. ${ }^{21}$ Similarly, in patients with facial nerve palsy (grade IV HB and above), gold plate implantation in upper eyelid should be considered to prevent corneal damage. ${ }^{22}$

\section{Conclusion}

With advances in radiotherapy, the role of surgery in skull base PG is being increasingly overlooked under the pretext that tumor control with preservation of function is adequate. However, in cases where total to near-total tumor clearance can be achieved with a minimal functional deficit, surgery should be preferred to give the patient a tumor-free survival. Handling of these cases requires an individualized approach based on the factors including economic status, age, comorbidities, tumor type and stage, multicentricity, the functionality of the tumor, status of the cranial nerves, venous drainage of the brain, and the degree of carotid involvement. Multidisciplinary approach and use of modern instrumentation can help in reducing the morbidity and mortality associated with surgery. Role of surgery should always be 
emphasized in developing countries where modern techniques of radiotherapy are seldom available. Postoperative rehabilitation is also necessary to improve the quality of life of the patient.

\section{Conflictof Interest}

None declared.

\section{References}

1 Lee JH, Barich F, Karnell LH, et al; American College of Surgeons Commission on Cancer; American Cancer Society. National Cancer Database report on malignant paragangliomas of the head and neck. Cancer 2002;94(3):730-737

2 Szymańska A, Szymański M, Czekajska-Chehab E, Gołąbek W, Szczerbo-Trojanowska M. Diagnosis and management of multiple paragangliomas of the head and neck. Eur Arch Otorhinolaryngol 2015;272(8):1991-1999

3 Burnichon N, Abermil N, Buffet A, Favier J, Gimenez-Roqueplo AP. The genetics of paragangliomas. Eur Ann Otorhinolaryngol Head Neck Dis 2012;129(6):315-318

4 Rothberg AS. Glomus tumors. J Am Med Assoc 1936; 107(7):520

5 Shin SH, Sivalingam S, De Donato G, Falcioni M, Piazza P, Sanna M. Vertebral artery involvement by tympanojugular paragangliomas: management and outcomes with a proposed addition to the fisch classification. Audiol Neurotol 2012;17(2):92-104

6 Shamblin WR, ReMine WH, Sheps SG. Harrison EG Jr. Carotid body tumor (chemodectoma). Clinicopathologic analysis of ninety cases. Am J Surg 1971;122(6):732-739

7 Pellitteri PK, Rinaldo A, Myssiorek D, et al. Paragangliomas of the head and neck. Oral Oncol 2004;40(6):563-575

8 Taïeb D, Kaliski A, Boedeker CC, et al. Current approaches and recent developments in the management of head and neck paragangliomas. Endocr Rev 2014;35(5):795-819

9 Sanna M, Flanagan S, Shin S, Mancini F, Piazza P, Introduction. In: Mario S, Paolo P. Seung-Ho S, Sean F, Fernando M, eds. Microsurgery of Skull Base Paragangliomas. New York: Thieme; 2013
10 House JW, Brackmann DE. Facial nerve grading system. Otolaryngol Head Neck Surg 1985;93(2):146-147

11 Bartels LJ, Gurucharri M. Pediatric glomus tumors. Otolaryngol Head Neck Surg 1988;99(4):392-395

12 Forest JA III, Jackson CG, McGrew BM. Long-term control of surgically treated glomus tympanicum tumors. Otol Neurotol 2001;22(2):232-236

13 Spector GJ, Maisel RH, Ogura JH. Glomus tumors in the middle ear. I. An analysis of 46 patients. Laryngoscope 1973;83(10):1652-1672

14 Jackson CG, Welling DB, Chironis P. Glasscock ME III, Woods CI. Glomus tympanicum tumors: contemporary concepts in conservation surgery. Laryngoscope 1989;99(9):875-884

15 Suárez C, Rodrigo JP, Bödeker CC, et al. Jugular and vagal paragangliomas: systematic study of management with surgery and radiotherapy. Head Neck 2013;35(8):1195-1204

16 Al-Mefty O, Teixeira A. Complex tumors of the glomus jugulare: criteria, treatment, and outcome. J Neurosurg 2002; 97(6):1356-1366

17 Makiese O, Chibbaro S, Marsella M, Tran Ba Huy P, George B. Jugular foramen paragangliomas: management, outcome and avoidance of complications in a series of 75 cases. Neurosurg Rev 2012;35(2):185-194, discussion 194

18 Sanna M, Jain Y, De Donato G, Rohit, Lauda L, Taibah A. Management of jugular paragangliomas: the Gruppo Otologico experience. Otol Neurotol 2004;25(5):797-804

19 Wanna GB, Sweeney AD, Carlson ML, et al. Subtotal resection for management of large jugular paragangliomas with functional lower cranial nerves. Otolaryngol Head Neck Surg 2014;151(6):991-995

20 Ivan ME, Sughrue ME, Clark AJ, et al. A meta-analysis of tumor control rates and treatment-related morbidity for patients with glomus jugulare tumors. J Neurosurg 2011;114(5):1299-1305

21 Thakar A, Sikka K, Verma R, Preetam C. Cricothyroid approximation for voice and swallowing rehabilitation of high vagal paralysis secondary to skull base neoplasms. Eur Arch Otorhinolaryngol 2011;268(11):1611-1616

22 Thurnher D, Novak CB, Neligan PC, Gullane PJ. Reconstruction of lateral skull base defects after tumor ablation. Skull Base 2007;17(1):79-88 